\title{
A case of Castleman disease that improved after kidney transplantation
}

\author{
Hee Ryong Lee', Jung Myung $\mathrm{An}^{1}$, Dong Ryeol Lee', Hyun Wook Choi ${ }^{2}$, \\ Joon Seok $\mathrm{Oh}^{3}$, Joong Kyung $\mathrm{Kim}^{3}$ \\ Departments of ${ }^{1}$ Nephrology and ${ }^{2}$ Radiology, Maryknoll Hospital, Busan, Korea; \\ ${ }^{3}$ Department of Nephrology, Bongseng Memorial Hospital, Busan, Korea
}

This is a case of a 56-year-old man with Castleman disease (CD) who improved after kidney transplantation (KTP). CD is an uncommon lymphoproliferative disorder that was found incidentally on biopsy during dialysis in the current patient and was followed up without further treatment. However, the lesion showed improvement after KTP. Therefore, active KTP can be considered even if $\mathrm{CD}$ is one of the lymphoproliferative disorders that can occur as a complication after KTP.

Keywords: Castleman disease; Kidney transplantation; Hemodialysis

\section{INTRODUCTION}

Castleman disease (CD), characterized as either unicentric $\mathrm{CD}$ (UCD) or multicentric $\mathrm{CD}$ (MCD), is an uncommon lymphoproliferative disorder with unclear underlying mechanisms [1]. It most frequently develops at the mediastinum (67\%) and less commonly at the retroperitoneal cavity (12\%) [2]. Transplantation-related CDs have been reported in the literature. There was a case report on a patient with mediastinal mass associated with paraneoplastic pemphigus and bronchiolitis obliterans who was diagnosed with $\mathrm{CD}$ and thus required pulmonary transplantation [3]. Several reports have demonstrated that human herpesvirus-8 (HHV-8) and Epstein-Barr virus (EBV)-associated monotypic large B-cell lymphoproliferative disorders are associated with mixed-variant $\mathrm{CD}$ after kidney transplantation (KTP). A case of CD after KTP was

Received September 27, 2018

Revised February 27, 2019

Accepted March 6, 2019

Correspondence to: Joon Seok Oh

Department of Nephrology, Bongseng Memorial Hospital, 401 Jungang-daero, Dong-gu, Busan 48775, Korea

Tel: +82-51-664-4000, Fax: +82-51-631-8054

E-mail: j-seok@hanmail.net also reportedly resolved after graft nephrectomy [4]. In addition, a Japanese KTP recipient recovering from end-stage renal failure due to $\mathrm{MCD}$ was also reported [5]. Herein, we report a Korean case of CD that improved after KTP.

\section{CASE REPORT}

A 56-year-old man with hypertensive nephropathy was undergoing hemodialysis (HD) since 2008. On December 28, 2011, the fourth year of $\mathrm{HD}$ treatment, he complained of abdominal discomfort. The physical examination showed no specific findings. Meanwhile, his laboratory findings showed a serum creatinine $(\mathrm{Cr})$ level of $8.9 \mathrm{mg} / \mathrm{dL}$, blood urea nitrogen of $49.1 \mathrm{mg} / \mathrm{dL}$, C-reactive protein (CRP) of $9.85 \mathrm{mg} / \mathrm{dL}$, lactate dehydrogenase of $115 \mathrm{IU} / \mathrm{L}$, hemoglobin of $9.0 \mathrm{~g} / \mathrm{dL}$, platelet count of $262,000 / \mathrm{mm}^{3}$, and serum albumin of $3.4 \mathrm{~g} / \mathrm{dL}$. Chest X-ray results did not show any specific findings. An abdominopelvic computed tomography (CT) scan revealed multiple, variable-sized, and homogeneously enhanced nodular densities in the mesenteric, retrocaval, paraaortic, and bilateral inguinal regions (sizes: up to $15 \mathrm{~mm}$ in the left paraaortic and $22 \mathrm{~mm}$ in the left inguinal region) (Fig. 1). Subsequently, a left in- 

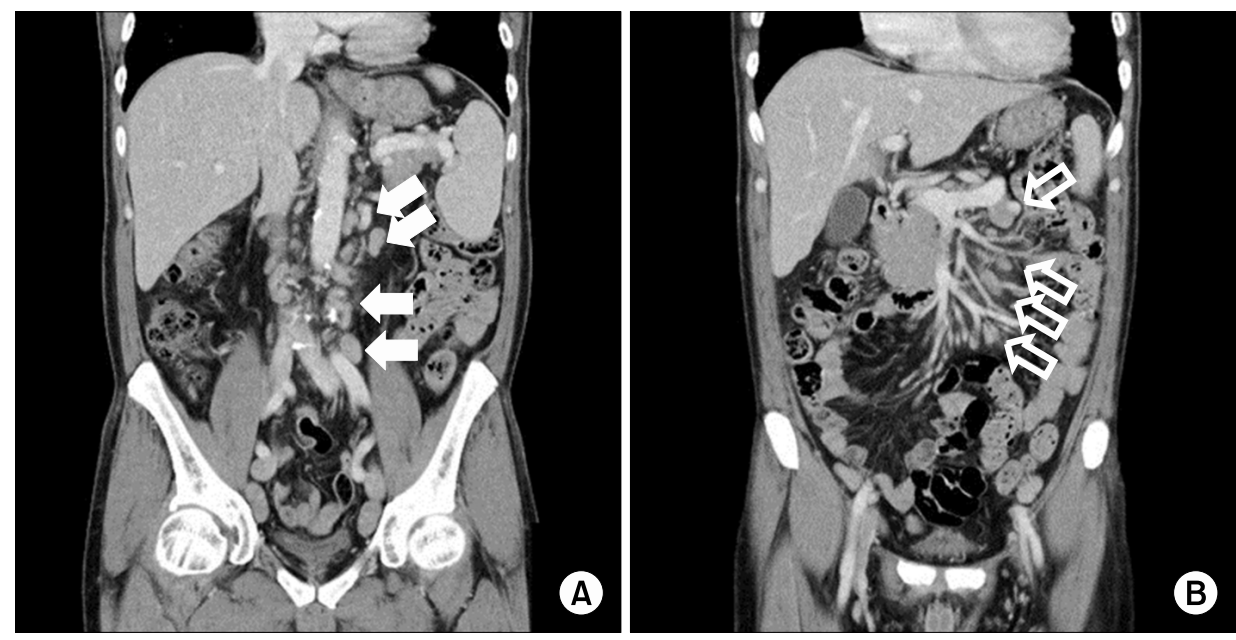

Fig. 1. Abdominopelvic computed tomography scan showed multiple, variable-sized, and homogeneously enhanced nodular densities in the paraaortic (A, solid arrows) and mesenteric (B, open arrows) regions. Although not shown here, retrocaval and inguinal regions were also observed.
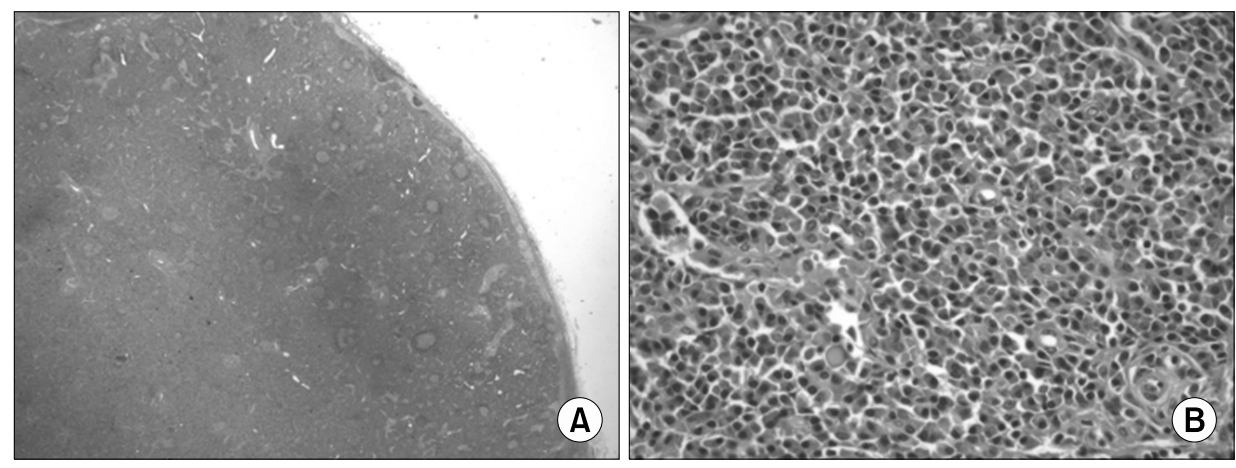

Fig. 2. Light-microscopy inguinal biopsy findings (H\&E). (A) The follicle size varied due to abnormally large germinal centers $(\times 10)$. (B) The interfollicular region was hypervascular and contained plasma cell sheets. Increased vascularity was observed in high-endothelial venules located at interfollicular zones $(\times 200)$.

guinal lymph node excisional biopsy was performed on January 9, 2012 (Fig. 2). The specimen consisted of a soft-tissue mass measuring $4.5 \times 4 \times 1.5 \mathrm{~cm}$. The cut surface revealed enlarged multiple lymph nodes (largest node size, $4 \times 2 \mathrm{~cm}$ cross-sectionally). The tissue was submitted for light microscopy, and paraffin sections were stained with hematoxylin and eosin [6]. Pathologically, the mass was diagnosed as $\mathrm{CD}$, plasma-cell type. Therefore, $\mathrm{HD}$ was performed, which alleviated his chief complaint after the supportive care. No CD treatment was performed, as no evidence of relapse was observed after symptom recovery. The patient underwent deceased-donor KTP on March 9, 2017. The preoperative abdominopelvic CT scan revealed persistent lymphadenopathy. This represented no significant difference, which is inconsistent with that of the previous CT scan findings (Fig. 3A). On chest CT scan, bilateral hilar and mediastinal lymph nodes and bilateral axillary lymph nodes, previously unverified, were identified (Fig. 4A).
The patient's panel reactive antibody results were 19\% in class I and 9\% in class II, but no donor-specific antibody was detected. Human leukocyte antigen mismatch $4 / 6$ (recipient: A 2/24, B 35/54, DR 14/15; donor: A 2/24, B 27/44, DR 1/7) was observed. Postoperative immunosuppressive therapy consisted of a triple-drug regimen: tacrolimus at a starting dose of $0.075 \mathrm{mg} / \mathrm{kg} /$ day and adjusted when the blood trough level was $>10 \mathrm{ng} / \mathrm{mL}$; mycophenolate mofetil $1,500 \mathrm{mg} /$ day, and a 2-month tapered dose of prednisolone from 250 to $5 \mathrm{mg} /$ day. Basiliximab $20 \mathrm{mg}$ was administered on the operation day and 4 days after KTP.

Intraoperatively, excisional biopsy was simultaneously performed on the lymph nodes located in the external iliac area. The specimens included two enlarged lymph nodes measuring $3 \times 2 \times 0.9 \mathrm{~cm}$ and $3.5 \times 1.5 \times 1 \mathrm{~cm}$. The histologic examination revealed plasma-cell-type $\mathrm{CD}$ showing histopathological findings similar to the previous biopsy results and no malignant progression. EBV im- 

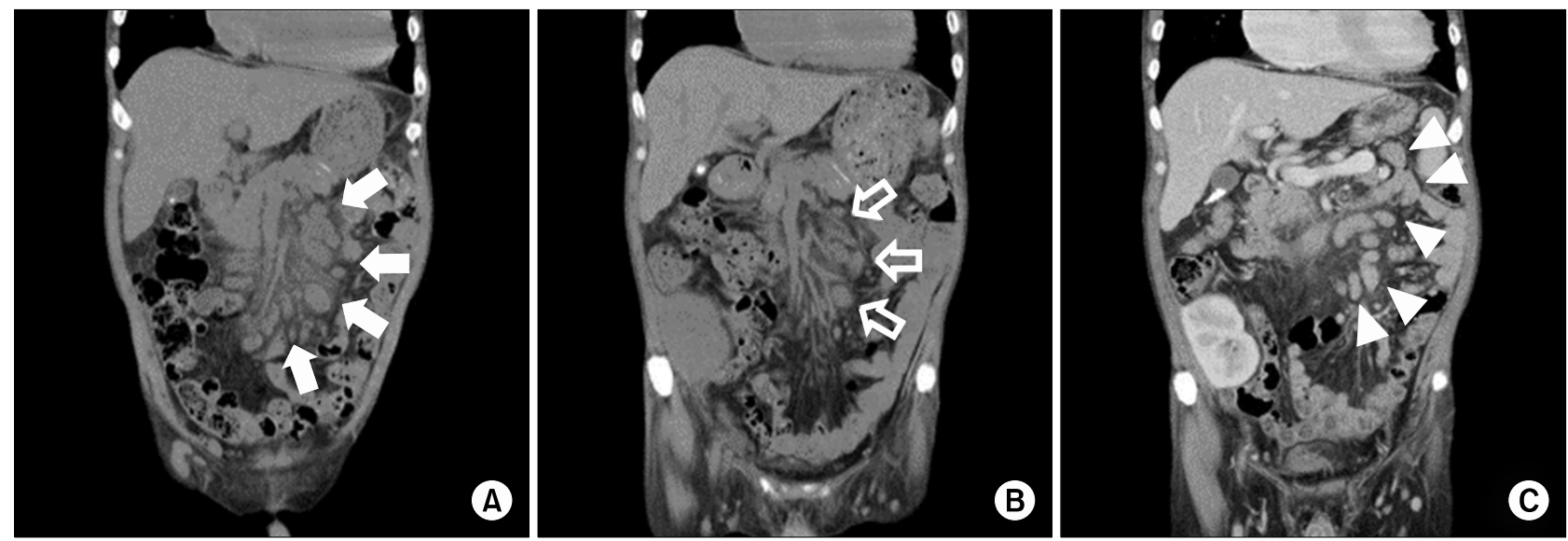

Fig. 3. (A) Abdominopelvic computed tomography (CT) scan before kidney transplantation. Compared with the previous CT scan (Fig. 1), the size became larger (solid arrows). (B) CT scan conducted 3 weeks after kidney transplantation showed a decreased lymph-node size compared with the previous CT scan (open arrows). (C) CT scan conducted 1 year after kidney transplantation showed that the size and number of lymph nodes remained unchanged (arrowheads).
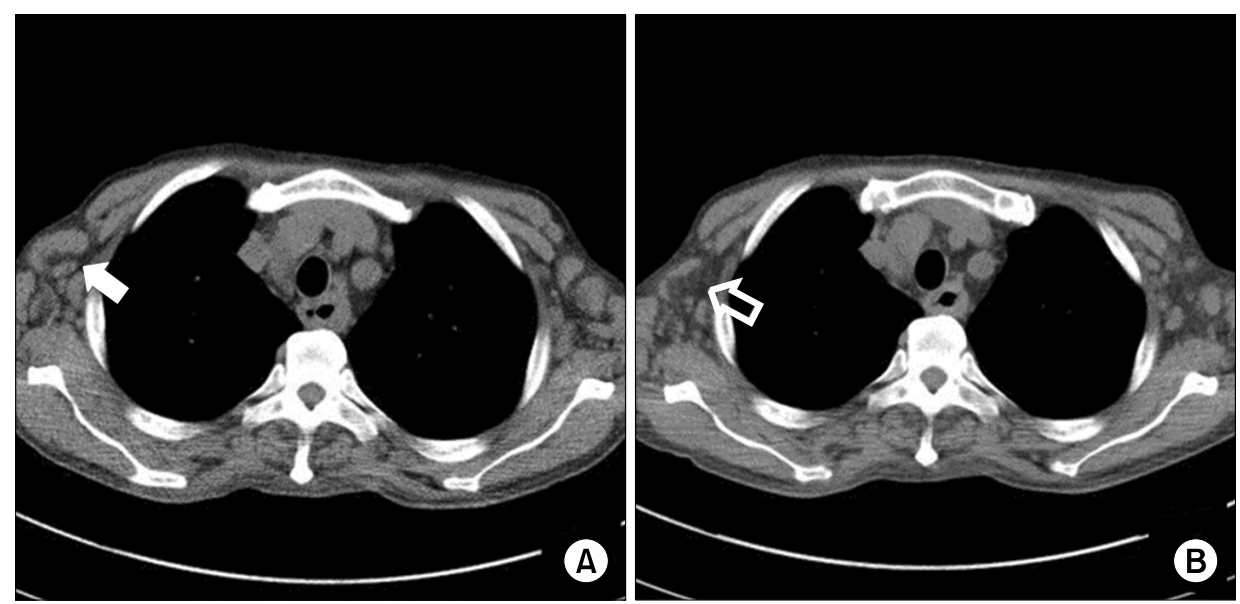

Fig. 4. (A) Chest computed tomography (CT) scan before kidney transplantation. Multiple enlarged mediastinal and hilar lymph nodes were detected, especially in the axillary lymph nodes (solid arrow). (B) The lymph node size in the CT scan at 3 weeks after kidney transplantation was smaller than that in the previous CT scan (open arrow) munoglobulin $\mathrm{G}$ (IgG)-positive and IgM-negative findings were also assessed, and HHV-8-negative results were found. Repeat CT scan was performed 3 weeks postoperatively, on March 31, 2017. Compared with the CT scan taken during KTP, the lymph nodes decreased in size (Figs. 3B, 4B). One year after KTP, the patient has normal graft function (serum $\mathrm{Cr}$ of approximately $1.4 \mathrm{mg} / \mathrm{dL}$ ) without clinical or pathological evidence of rejection. In addition, surveillance with radiologic (Fig. 3C) and hematologic follow-up demonstrated no evidence of $\mathrm{CD}$ aggravation.

\section{DISCUSSION}

$\mathrm{CD}$ is classified into at least three distinct disorders based on the number of regions of enlarged lymph nodes with characteristic histopathologic features and presence/absence of HHV-8 (also known as Kaposi-sarcoma-associated herpesvirus) infection [7-9]. UCD involves one or more enlarged lymph nodes in a single region of the body, whereas MCD involves multiple regions of lymphadenopathy. MCD is categorized based on HHV-8. HHV-8-negative idiopathic MCD (iMCD) has at least three subgroups: polyneuropathy, organomegaly, endocrinopathy, monoclonal protein, skin changes (POEMS)-associated; thrombocytopenia, anasarca, fever, reticulin fibrosis, and organomegaly 
(TAFRO) syndrome; and not otherwise specified (NOS) $[10,11]$. The patient was classified as having HHV-8-negative iMCD owing to lymphadenopathy in multiple regions and HHV-8-negative status. Moreover, as he did not have POEMS syndrome or the TAFRO subtype, he was classified as having iMCD-NOS.

The etiology of iMCD is unknown; however, possible mechanisms have been suggested to include autoimmune, autoinflammatory, neoplastic, and infectious processes $[12,13]$. Interleukin (IL)-6 is an essential and sufficient factor for its symptomatology, histopathology, and pathogenesis. IL-6 is a multifunctional cytokine characterized by plasmacytosis, hypergammaglobulinemia, thrombocytosis, acutephase protein production, and activation of macrophages and $\mathrm{T}$ cells [14]. In addition to IL-6, vascular endothelial growth factor $\mathrm{IL}-1$ is also involved in the etiology of $\mathrm{MCD}$ $[15,16]$. In this patient, the autoantibodies were negative, and none of the clinical pathological features of autoimmune disease were found. In fact, notwithstanding the fact that these features are typically observed in lymphoma, they were not evident in our patient. However, no infections caused by pathogens other than HHV-8 were identified either. In addition, the mechanism of germline mutations in genes regulating the inflammation was not confirmed. Therefore, either an infectious or an autoinflammatory mechanism was suggested to be involved.

The following two major criteria must be satisfied to diagnose iMCD: enlarged lymph nodes $(\geq 1 \mathrm{~cm}$ in the short-axis diameter), $\geq 2$ lymph-node stations, and histopathologic lymph-node features [17]. The iMCD spectrum is characterized by regressed/atrophic/atretic germinal centers, follicular dendritic cell prominence, vascularity, polytypic plasmacytosis in the interfollicular space, and hyperplastic germinal centers. iMCD is also diagnosed when at least two of the 11 minor criteria were found, with at least one of them as a laboratory criterion. The minor criteria consist of laboratory and clinical data. The minor laboratory criteria include elevated CRP or erythrocyte sedimentation rate, anemia, thrombocytopenia or thrombocytosis, hypoalbuminemia, renal dysfunction or proteinuria, and polyclonal hypergammaglobulinemia. The minor clinical criteria include constitutional symptoms (night sweats, fever, weight loss, or fatigue), enlarged spleen and/or liver, fluid accumulation, eruptive cherry hemangiomatosis or violaceous papules, and lymphocytic interstitial pneumonitis. However, even if these criteria are all met, various infection-related disorders, autoimmune/ autoinflammatory diseases, and malignant/lymphoproliferative disorders that may be confused as iMCD should still be excluded.

The natural history of HHV-8-negative iMCD also varies. The prognosis of untreated MCD is poor. The 5-year overall survival ranges from $55 \%$ to $77 \%[18,19]$. However, the indolent form, rather than the rapidly progressive form, does not seem to deteriorate with time. Disease severity should be assessed by determining the direction of $\mathrm{MMCD}$ treatment [20]. In non-severe diseases without life-threatening organ failure, an anti-IL-6 monoclonal antibody, siltuximab, or an anti-IL-6 receptor monoclonal antibody, tocilizumab, is the primary iMCD treatment. Siltuximab (11 $\mathrm{mg} / \mathrm{kg}$ intravenous infusion every 3 weeks for 12 weeks) has been evaluated in a double-blind, placebo-controlled, randomized study using a control arm of best supportive care with up to $60 \mathrm{mg}$ of prednisone. The combined long-term symptomatic and tumor response was 34\% [21]. A single-arm study on 28 Japanese patients receiving tocilizumab $(8 \mathrm{mg} / \mathrm{kg}$ intravenously every 2 weeks for 16 weeks) demonstrated a high response rate in terms of symptoms, laboratory parameters, and reduction of lymphadenopathy [22]. Even if a sufficient response after treatment is detected, the IL-6 inhibitor should be maintained to prevent symptom recurrence when the treatment is stopped (remission is defined as the absence of abnormalities on physical examinations and laboratory or imaging studies). In response to insufficient response or progressive organ failure during response access, systemic chemotherapy with or without an immunomodulator/immunosuppressant or rituximab may be considered [23]. Patients with severe $\mathrm{MMCD}$ who have marked organ dysfunction, poor performance status, and/or require critical care should be promptly started on a high-dose steroid regimen (e.g., methylprednisolone $500 \mathrm{mg}$ daily) together with siltuximab or tocilizumab. However, POEMS-associated iMCD does not respond to IL-6 inhibitors; therefore, hematopoietic cell transplantation and radiation treatment can be considered [20]. The present patient was 
not treated with IL-6 inhibitors during KTP. However, glucocorticoids used during KTP relieved the symptoms by inhibiting hypercytokinemia [23], and tacrolimus prevented $\mathrm{CD}$ deterioration by inhibiting $\mathrm{T}$ helper 1 cell inflammation, which is considered as one of the $\mathrm{CD}$ pathologies [24].

Active malignancy is an absolute contraindication for KTP. Patients with posttransplant lymphoproliferative disorder (PTLD) should not receive a second transplant until at least 2 years after a successful treatment [25]. A lymphoproliferative disorder such as $\mathrm{CD}$ may be found as a posttransplant complication. Determining an indication for KTP is difficult in a potentially systemic disease such as MCD. However, some reports demonstrated that $\mathrm{CD}$ does not deteriorate after KTP. A case of hyaline vascular-type CD incidentally found during KTP showed no major complications at the 3-year follow-up [26]. There is also a case report of CD recovery after lung transplantation [27]. In our case, either active CD or PTLD was rarely detected, and no clear contraindication was evident. If symptoms of MCD are maintained without deterioration, as in the present case, transplantations can be performed successfully.

In conclusion, an $\mathrm{MMCD}$ patient who underwent $\mathrm{HD}$ for end-stage renal disease was successfully managed with KTP, without deterioration or progression of $\mathrm{CD}$. In this case, CD did not prevent KTP; therefore, more active KTPs should be recommended for $\mathrm{CD}$ patients based on this finding.

\section{CONFLICT OF INTEREST}

No potential conflict of interest relevant to this article was reported.

\section{ORCID}

Hee Ryong Lee https://orcid.org/0000-0003-3978-8598

Dong Ryeol Lee https://orcid.org/0000-0002-7194-6202

Joong Kyung Kim https://orcid.org/0000-0002-8907-3251

\section{REFERENCES}

1. Soumerai JD, Sohani AR, Abramson JS. Diagnosis and man- agement of Castleman disease. Cancer Control 2014;21: 266-78.

2. Otto M, Wieprzowski L, Dzwonkowski J, ZiarkiewiczWróblewska B. Castleman's disease: an unusual indication for laparoscopic adrenalectomy. Wideochir Inne Tech Maloinwazyjne 2012;7:50-4.

3. Chin AC, Stich D, White FV, Radhakrishnan J, Holterman MJ. Paraneoplastic pemphigus and bronchiolitis obliterans associated with a mediastinal mass: a rare case of Castleman's disease with respiratory failure requiring lung transplantation. J Pediatr Surg 2001;36:E22.

4. Al Otaibi T, Al Sagheir A, Ludwin D, Meyer R. Post renal transplant Castleman's disease resolved after graft nephrectomy: a case report. Transplant Proc 2007;39:1276-7.

5. Murakami K, Kobayashi T, Okubo K, Kamba T, Yoshimura $\mathrm{K}$, Ogawa O. Successful renal transplantation for end-stage renal insufficiency developed in a patient with Castleman's disease. Transpl Int 2013;26:e61-2.

6. Brodsky SV, Albawardi A, Satoskar AA, Nadasdy G, Nadasdy T. When one plus one equals more than two: a novel stain for renal biopsies is a combination of two classical stains. Histol Histopathol 2010;25:1379-83.

7. Castleman B, Towne VW. Case records of the Massachusetts General Hospital: Case No. 40231. N Engl J Med 1954; 250:1001-5.

8. Castleman B, Iverson L, Menendez VP. Localized mediastinal lymphnode hyperplasia resembling thymoma. Cancer 1956;9:822-30.

9. Martin JM, Bell B, Ruether BA. Giant lymph node hyperplasia (Castleman's disease) of hyaline vascular type: clinical heterogeneity with immunohistologic uniformity. Am J Clin Pathol 1985;84:439-46.

10. Dispenzieri A. POEMS syndrome and Castleman's disease. In: Zimmerman TM, Kumar SK, eds. Biology and management of unusual plasma cell dyscrasias. New York, NY: Springer; 2017. p. 41.

11. Iwaki N, Fajgenbaum DC, Nabel CS, Gion Y, Kondo E, Kawano M, et al. Clinicopathologic analysis of TAFRO syndrome demonstrates a distinct subtype of HHV-8-negative multicentric Castleman disease. Am J Hematol 2016;91: 220-6.

12. Kojima M, Motoori T, Asano S, Nakamura S. Histological diversity of reactive and atypical proliferative lymph node lesions in systemic lupus erythematosus patients. Pathol Res Pract 2007;203:423-31.

13. Kojima M, Motoori T, Nakamura S. Benign, atypical and malignant lymphoproliferative disorders in rheumatoid arthritis patients. Biomed Pharmacother 2006;60:663-72.

14. Kishimoto T. IL-6: from its discovery to clinical applications. 
Int Immunol 2010;22:347-52.

15. Fajgenbaum DC, Rosenbach M, van Rhee F, Nasir A, Reutter J. Eruptive cherry hemangiomatosis associated with multicentric Castleman disease: a case report and diagnostic clue. JAMA Dermatol 2013;149:204-8.

16. El-Osta H, Janku F, Kurzrock R. Successful treatment of Castleman's disease with interleukin-1 receptor antagonist (Anakinra). Mol Cancer Ther 2010;9:1485-8.

17. Fajgenbaum DC, Uldrick TS, Bagg A, Frank D, Wu D, Srkalovic G, et al. International, evidence-based consensus diagnostic criteria for HHV-8-negative/idiopathic multicentric Castleman disease. Blood 2017;129:1646-57.

18. Shin DY, Jeon YK, Hong YS, Kim TM, Lee SH, Kim DW, et al. Clinical dissection of multicentric Castleman disease. Leuk Lymphoma 2011;52:1517-22.

19. Dispenzieri A, Armitage JO, Loe MJ, Geyer SM, Allred J, Camoriano JK, et al. The clinical spectrum of Castleman's disease. Am J Hematol 2012;87:997-1002.

20. van Rhee F, Voorhees P, Dispenzieri A, Fosså A, Srkalovic $\mathrm{G}$, Ide $\mathrm{M}$, et al. International, evidence-based consensus treatment guidelines for idiopathic multicentric Castleman disease. Blood 2018;132:2115-24.

21. Nishimoto N, Sasai M, Shima Y, Nakagawa M, Matsumoto $\mathrm{T}$, Shirai T, et al. Improvement in Castleman's disease by humanized anti-interleukin-6 receptor antibody therapy. Blood 2000;95:56-61.

22. Nishimoto N, Kanakura Y, Aozasa K, Johkoh T, Nakamura M, Nakano S, et al. Humanized anti-interleukin-6 receptor antibody treatment of multicentric Castleman disease. Blood 2005;106:2627-32.

23. Liu AY, Nabel CS, Finkelman BS, Ruth JR, Kurzrock R, van Rhee F, et al. Idiopathic multicentric Castleman's disease: a systematic literature review. Lancet Haematol 2016; 3:e163-75.

24. Shirai T, Onishi A, Waki D, Saegusa J, Morinobu A. Successful treatment with tacrolimus in TAFRO syndrome: two case reports and literature review. Medicine (Baltimore) 2018; 97:e11045.

25. Stallone G, Infante B, Grandaliano G. Management and prevention of post-transplant malignancies in kidney transplant recipients. Clin Kidney J 2015;8:637-44.

26. Yousif ME, El Hassan AM, Abdulrahim AS. Castleman's disease in a kidney failure patient diagnosed incidentally during transplantation. Arab J Nephrol Transplant 2011;4:31-3.

27. Morimura Y, Chen F, Kinjo T, Miyagawa-Hayashino A, Kubo T, Yamada T, et al. Successful single-lung transplantation for multicentric Castleman disease. Ann Thorac Surg 2014;98:e63-5. 